\title{
TASAS DE INTERÉS REAL NEUTRALES Y LAS NORMAS INTERNACIONALES DE INFORMACIÓN FINANCIERA
}

\author{
REAL NEUTRAL INTEREST RATE AND INTERNATIONAL FINANCIAL \\ REPORTING STANDARDS
}

\author{
Raúl Alberto Arrarte Mera \\ Doctor en Ciencias Contables y Empresariales - Docente de la Facultad de Ciencias Contables - Universidad Nacional Mayor de \\ San Marcos, Lima, Perú - Email: rarrartem@unmsm.edu.pe (Autor Corresponsal)
}

[Recibido: 25/05/2016 Aceptado: 10/08/2017]

\begin{abstract}
RESUMEN
La presente investigación es del tipo exploratorio-descriptivo-cuantitativa basado en la construcción de flujos creciente y decreciente de ingresos futuros a tasas bajas y altas de interés, esto es, una simulación matemática. Los objetivos son: Evaluar cómo instaurar un factor de descuento neutral, a nivel de América Latina y el Caribe, para aplicar a las normas internacionales de información financiera. Verificar cómo estimar una tasa de interés real neutral. Estudiar si una tasa de interés real neutral normada y transparente coadyuva al combate contra el lavado de activos y la corrupción. Se concluye que, tanto en los países emergente y no emergente, se premia el uso eficiente de los instrumentos macroeconómicos con bajas tasas de interés, por lo contrario, elevadas tasas de interés a los países con superiores índices de inflación por manejo defectuoso de su economía. Confirmamos que es conveniente para nuestros países que el Banco Central de Reserva del Perú, y demás entidades afines de América Latina y el Caribe, normalicen trimestralmente las tasas de interés real neutrales a nivel regional, utilizando las dos lenguas oficiales de la Organización de las Naciones Unidas (ONU): español e inglés; aplicando criterios mundiales de instituciones reconocidas como: Reserva Federal de Estados Unidos de Norte América (FED), Bancos Centrales de Reserva de cada país y calificadora de riesgos J.P. Morgan Chase de New York; en base a una canasta de instrumentos como: tasa básica FED, tasas de inflación y/o devaluación, riesgo país, y costo administrativo del dinero.
\end{abstract}

\section{PALABRAS CLAVE}

Devaluación, factor de descuento, inflación, riesgo país, tasa de interés, valor futuro.

\begin{abstract}
The present investigation is an exploratory-descriptive-quantitative type based on the construction of increasing and decreasing flows of future income at low and high interest rates, that is, a mathematical simulation. The objectives were to evaluate a way to establish a neutral discount factor, in Latin America and the Caribbean, to apply to international financial reporting standards, to verify an estimation of a neutral real interest rate and to study whether a regulated and transparent neutral real interest rate contributes to the fight against money laundering and corruption. It is concluded that, in both emerging and non-emerging countries, the efficient use of macroeconomic instruments with low interest rates is rewarded, on the contrary, high interest rates are given to countries with higher inflation rates due to faulty management of their economy. We confirm that it is convenient for our countries that the Central Reserve Bank of Peru and other related entities in Latin America and the Caribbean, quarterly normalize neutral real interest rates at the regional level, using the two official languages of the United Nations (UN): Spanish and English; applying global criteria from recognized institutions such as the Federal Reserve of the United States of America (FED), Central Reserve Banks of each country and the risk assessor J.P. Morgan Chase of New York; based on a set of instruments such as the basic EDF rate, inflation and / or devaluation rates, country risk, and administrative cost of money.
\end{abstract}

\section{KEYWORDS}

Devaluation, discount factor, inflation, country risk, interest rate, future value. 


\section{INTRODUCCIÓN}

¿Por qué se ha realizado esta investigación? Por el interés que despierta en el contexto científico contable latinoamericano la discusión técnica de determinar el costo del dinero o tasa de interés, que hacen referencia al factor de descuento que debe utilizarse para calcular estimaciones de flujos de efectivo futuros promovido por la Norma Internacional de Contabilidad No. 19, (NIC), Beneficios a los Empleados, para el cálculo de beneficios a largo plazo; la NIC No. 36, Deterioro del Valor de los Activos, para determinar el valor de uso del activo; la NIC No. 37, Provisiones, Pasivos Contingentes y Activos Contingentes, para aplicar al valor presente de los desembolsos; y la Norma Internacional de Información Financiera No. 13 (NIIF), Medición del Valor Razonable, para el cálculo de las variables de nivel 3; que emite la Junta de Estándares Internacionales de Contabilidad (IASB o International Accounting Standards Board). La pregunta es ¿Por qué el Banco Central de Reserva del Perú (BCRP), y demás entidades afines de América Latina y el Caribe no publican trimestralmente una tasa de interés real neutral normalizada para efectos del cumplimiento de las normas contables arriba citadas, discriminada por países emergentes y no emergentes? Porque hoy se conoce el contenido de una tasa de interés donde participan instituciones del mundo y que solamente debemos normarla en base a criterios públicos generales que determinen pautas mínimas de control emitidas por organizaciones como: la FED o Reserva Federal de los Estados Unidos de Norteamérica; los Bancos Centrales de Reservas de los países del globo; el Banco J.P. Morgan Chase, calificadora de riesgo mundial, y supervisada por los Contadores Públicos de América Latina y el Caribe.
Así se zanjaría el problema de valuación aplicando una tasa neutral de costo de capital internacional debidamente normada. Permitiendo repartir entre los accionistas utilidades efectivamente generadas y presentar estados financieros confiables consecuentes con la economía real y no imaginaria. Evitando a futuro el abuso del valor razonable y su descrédito consiguiente.

Antes de continuar con el desarrollo de estas ideas, es necesario precisar conceptos sobre qué es una tasa de interés desde el punto de vista de las normas internacionales de información financiera. No es igual decir costo de capital y costo de oportunidad. El primero está referido al costo del dinero en el mercado, al capital ajeno, a los préstamos bancarios. Mientras que el segundo, es la tasa de rentabilidad que desea el empresario, o también denominado "costo de oportunidad", proveniente del capital propio. Por tanto, al referirnos al factor descuento en el caso particular de calcular el deterioro de los activos tangibles e intangibles, nos estamos refiriendo al costo del dinero en el mercado, a las tasas de interés que cobran o fijan los bancos comerciales. Por tanto, la tasa de interés que establece un banco tiene dos componentes incluidos: el tipo de interés libre de riesgo + compensación por riesgo. No es correcto agregar a esta tasa un nuevo factor de riesgo, porque la tasa de mercado ya tiene incluido su factor de riesgo. Tampoco es correcto utilizar el modelo del costo del capital promedio ponderado conocido como WACC (Weighted Average Cost of Capital), porque representa el promedio de todas las fuentes de fondos, (tanto capital ajeno como propio), identificado en un momento determinado en el tiempo de la empresa en marcha. En concreto: una cosa es calcular la rentabilidad de un proyecto, que incluye el costo de sus fuentes de financiamiento (capital propio y capital ajeno), y otra, muy distinta, el deterioro de un activo tangible o intangible en base al costo de capital."El concepto de tasa neutral de interés es mencionada en los escritos del economista sueco Knut Wicksell. Para él la tasa neutral es aquella que equilibra, el pleno empleo, el mercado del ahorro y la inversión, la cual puede diferir de la tasa de interés cobrada y pagada por los bancos en el sistema financiero, la cual llama la tasa de mercado (Fuentes, J.R. 2008: 136)".

En este caso debemos aplicar una tasa de interés neutral proveniente de una canasta de instrumentos como: tasa básica, tasas de inflación y/o devaluación, riesgo país, y costo administrativo del dinero proveniente de instituciones reconocidas como: Reserva Federal de Estados Unidos de Norte América, Bancos Centrales de Reserva de cada país de América Latina y el Caribe, y calificadora de riesgos J.P. Morgan Chase de New York, indicadores que se publican mensualmente a nivel mundial.

Como es de notar el tema ha generado discusiones en todos los ámbitos económico-financieros del mundo, y en particular a nivel de los Contadores Públicos en calidad de auditores independientes que por ley tienen la delicada función de dar opinión sobre la situación de los estados financieros que se publican en las Bolsas de Valores en forma trimestral, y en los países del globo a fin de año, para efectos del cálculo del impuesto a la renta. Si aplicamos una tasa de descuento menor hay mínimas pérdida por deterioro y con máximas utilidades; y a los ingresos del Estado con mayores impuestos. Y, si la tasa de descuento es elevada, ocurre todo lo contrario. Consiguientemente para obviar esta 
discusión lo ideal está en el "justo medio".

Los objetivos, general y específicos, son los siguientes:

1. Evaluar cómo formar un factor de descuento neutral, a nivel de América Latina y el Caribe, para aplicar a las normas internacionales de información financiera.

2. Verificar cómo estimar una tasa de interés real neutral.

3. Estudiar si una tasa de interés real neutral normada y transparente coadyuva al combate contra el lavado de activos y la corrupción.

Este trabajo aspira a generar corriente de opinión e influenciar en el ambiente contable porque la tasa de interés neutral sea normada por los Bancos Centrales de Reserva de América Latina y el Caribe para el mundo regional dividida en países emergentes y no emergentes, asumiendo que es ya una realidad el modelo contable que se patrocina. Para ello se consideró necesario analizar el contenido de una tasa de interés, además de los valores que la influyen. En el marco teórico, se resumen opiniones técnicas del Banco Central del Uruguay, del Banco Central de Reserva del Perú, tesis de grado de Guatemala, Ecuador y España y casuística de profesionales peruanos; también se analizan las tasas de interés internacionales. Luego se examina la importancia que tiene el nivel de la tasa de interés con una simulación matemática. Y en la última parte se consignan las consideraciones finales sobre el tema analizado a modo de conclusión.

\section{Devaluación}

La devaluación es la disminución o pérdida del valor nominal de una moneda corriente frente a otras monedas extranjeras. En el caso de Perú, sería la reducción del valor del sol versus el dólar, el euro, la libra esterlina, el yen y en general cualquier moneda de otro país.

\section{Factor de descuento}

Es una fórmula matemática representada por el siguiente enunciado:

Factor de Descuento $=$ FD $=$ Valor actual de 1 sol.

$\mathrm{FD}=1 /(1+\mathrm{r})^{\mathrm{t}}$

Donde:

$\mathrm{r}=$ Tasa de interés

$\mathrm{t}=$ Tiempo

Los factores de descuento se utilizan para calcular el valor actual de cualquier flujo de caja.

\section{Inflación}

En general este fenómeno económico significa "incremento en el nivel general de precios de bienes y servicios" y/o "cualquier incremento en el valor de los activos". Un instrumento de política monetaria que utilizan los Bancos Centrales del mundo para controlarla es la denominada tasa de referencia (TR). A mayor o menor masa monetaria en poder de familias y empresas tiene efectos en el nivel de actividad económica, la tasa de inflación y el precio del dólar. Por lo contrario, mayor consumo e inversión tiene resultados directos en el crecimiento de la economía. Es obligación de los Bancos Centrales defender la estabilidad monetaria, y minimizar la inflación y el tipo de cambio.

Sin embargo, actualmente en el caso particular de Perú, por las secuelas que ha generado en la infraestruc- tura física del país el fenómeno del "Niño Costero", no es fácil para el Banco Central de Reserva del Perú, (BCRP), mantener la inflación en el rango meta de $1 \%$ a $3 \%$ anual. Así, por ejemplo, la inflación anualizada a marzo de 2017 llegó a 3,97\% por efectos del cambio climático que está sucediendo en el Perú, y ha generado intensas lluvias en zonas áridas y causado inundaciones en ciudades del norte del país como Trujillo, Chiclayo, Piura y Tumbes, cuyo costo de reconstrucción de ciudades y carreteras costaría alrededor de 12,000 millones de dólares. La disyuntiva del BCRP, es mantener o bajar su tasa de referencia de $4,25 \%$ a menos. Pero la Reserva Federal (FED), de EE.UU. También subió su tasa de interés de referencia y se anunció que habría tres subidas más en el año. Esto último restringe la política del BCRP, porque si baja su tasa la diferencia entre ambas aumenta, lo que genera el riesgo de que salgan o vengan menos capitales al Perú. Así, si el BCRP bajaba la tasa de interés para reactivar la economía sin tener en cuenta que la inflación tiene tendencia al alza y dejando de lado la política de la FED, el resultado hubiera sido peor. Por tanto, en esta coyuntura de desaceleración económica, es fundamental, para dinamizar la actividad empresarial, impulsar la inversión pública a través de asociaciones público-privadas (APP), y de obras por impuestos.

\section{Riesgo país}

Se define como una medida de la probabilidad que un país emergente incumpla las obligaciones financieras correspondientes a su deuda externa. Término asociado a Riesgo País es el de Riesgo Soberano, que da lugar a calificaciones crediticias (rating crediticio) que Empresas Calificadoras de Riesgo(ECR), internacionales elabo- 
ran respecto a las deudas públicas de los países. La finalidad de las ECR es dar una opinión como entidad independiente, especializada en el estudio de riesgo, sobre la calidad crediticia de la emisión de valores (Gorfinkiel y Lapitz, 2003:2).

En la práctica el riesgo país se mide con el EMBI (Emerging Market Bond Index), que fue creado por la firma internacional JP Morgan Chase y que da seguimiento diario a una serie de instrumentos de deuda de dólares emitidos por distintas entidades (Gobiernos, Bancos y Empresas), en países emergentes.

Si bien, existen distintas formas de medirlo, la más usada de ellas compara la tasa de interés de un bono de un país $\mathrm{X}$ (que tienen cierto nivel de riesgo) con la tasa de interés de un bono emitido por el Tesoro Americano (que se admite es un bono libre de riesgo). En principio se supone que el riesgo de que los bonos emitidos por Estados Unidos no sean pagados es muy baja (más baja que cualquier otra en el mundo) y por tanto su riesgo de no pago es casi cero.

Si este premio por la espera es igual para ambos bonos, entonces la diferencia entre las tasas de interés de los bonos del país X y los bonos estadounidenses reflejará el "riesgo" de invertir en el país X. Ejemplo: Como podemos ver, si la tasa de interés de un bono peruano es de $9,5 \%$, y la tasa de interés de un bono emitido por EEUU es de $5,0 \%$ se puede decir que el riesgo país del Perú es de 4,5\%. (9,5\% - 5,0\% $=4,5 \%)$. La cual se basa en la escala de 100 puntos básicos, equivalente a $1 \%$. En el caso del ejemplo, el 4,5\% de diferencia es equivalente a 450 puntos básicos. El riesgo país, como hemos mencionado, refleja las condiciones existentes en una economía emergente y sus vinculaciones con la econo- mía global. Se denomina emergente a todo país que por su poder económico y político son potenciales agentes de cambio en la escena internacional. Más conocidos como los países BRIC (Brasil, Rusia, India y China). $\mathrm{Y}$ actualmente más conocidos como los E7 (China, India, Brasil, Rusia, Indonesia, México y Turquía) cuyo producto bruto interno superará en el futuro a los G7 (Estados Unidos, Japón, Alemania, Reino Unido, Francia, Italia y Canadá).

\section{Tasa de Interés}

Económicamente hablando, la tasa de interés es el precio del dinero en el mercado financiero o el porcentaje que paga un capital invertido en un período de tiempo. La tasa de interés es fijada por el Banco Central de cada país a los otros bancos que, a su vez, la fijan a los sujetos de crédito por los préstamos otorgados.

Existen tipos de interés implícito de bonos gubernamentales a tasas de interés fijo o tasas de interés interbancario al que los bancos se prestan dinero ente sí. Precisa determinar la tasa de interés: tasa preferente (prime rate), tasa Libor, y/o la tasa de rendimiento ajustado por riesgo.

Generalmente, si hay suficiente liquidez en la economía, la tasa tiende a disminuir, en tanto que, si existe escasez de dinero, la tasa tiende a aumentar. Las tasas altas estimulan el ahorro y frenan la inflación ya que disminuye el consumo al incrementarse el costo de las deudas; no obstante, también frenan el crecimiento económico. Las tasas bajas, por el contrario, promueven el crecimiento económico ya que estimulan el consumo; sin embargo, pueden generar inflación al inyectar mayor circulante en la economía. Un tema de macroeconomía muy complejo y complicado.
Quizás la confusión en el uso del modelo está en que para determinar el valor de uso de un activo tangible o intangible existen dos pasos previos:

a) Definir el flujo de efectivo

b) Identificar la tasa de interés para calcular el factor de descuento

En el primer caso es correcto utilizar el modelo WACC o costo de capital promedio ponderado y en el segundo, sólo y únicamente, el costo de capital simple o tasa básica de interés bancaria vigente al momento de la decisión del cálculo del deterioro del activo. Que es el tipo legal del dinero y/o el tipo de redescuento del Banco Central de Reserva del Perú.

En un trabajo muy interesante Curti, D. (2010:14-15), funcionario del Banco Central del Uruguay, identifica tres vertientes de la literatura:

- Por la estructura de la industria bancaria, este enfoque surge de la vertiente de la organización industrial, la que enfatiza aspectos como la estructura del mercado, el nivel de concentración y competencia entre los bancos, barreras a la entrada, la existencia de bancos públicos y el nivel de desarrollo de los mercados financieros. En esta corriente se inscriben los trabajos de Hannan y Berger (1989 y 1991), Hannan (1991), Neumark y Sharpe (1992), Cottarelli y Kourelis (1994), Scholnick (1996), Wong (1997), Corvoisier y Gropp (2001), González y Salas (2005), Bikker, Sorensen, Van Leuvensteijn y Van Rixtel (2008).

- Por las características de los bancos, tales como tamaño del banco, tipo de clientes, tipo de fondeo y estructura del pasivo, preferencias por el riesgo, composición del por- 
tafolio, etc. Los trabajos de Angbazo (1997) Weth (2002), Berstein y Fuentes (2003) siguen esta línea de análisis.

- Por la existencia de información asimétrica entre los bancos y quienes toman créditos, esto genera los problemas de selección adversa y riesgo moral. El trabajo de Winker (1999) combina el racionamiento de crédito debido a información asimétrica con un enfoque de fijación de tasa de interés según el costo marginal, dado por la tasa de interés del mercado de dinero. A partir de esto se elabora un modelo que explica la lentitud del ajuste de las tasas de depósitos y de créditos ante cambios en la tasa del mercado de dinero debido al problema de selección adversa. Los trabajos de Lower y Rholing (1992) y Nabar, Park y Saunders (1993) también se inscriben en esta vertiente y explican la rigidez de las tasas de interés por el racionamiento de crédito debido a la selección adversa, los costos de cambio, el compartir riesgos y la irracionalidad de los consumidores.

Concluye: el régimen monetario y las condiciones del mercado monetario son factores que inciden en la formación de las tasas de interés bancarias, $y$, por otro lado, las condiciones financieras y las del mercado de crédito en particular, las cuales reflejan los riesgos que asumen los bancos en su rol de intermediarios financieros, son los otros factores relevantes en la formación de las tasas de interés bancarias.

Tasas de interés de fondos federales de Estados Unidos de Norteamérica

Es el precio que se paga por el dinero en el mercado interbancario norteamericano por préstamos a un día (overnight). Es una tasa de interés libre ya que no está regulada directamente por la Reserva Federal de Estados Unidos. Los bancos norteamericanos deben mantener un porcentaje de sus depósitos en la Reserva Federal para atender posibles episodios de salida de depósitos. Estos fondos o encajes son los denominados federal funds.

En un momento determinado algunos bancos tienen más fondos que los requeridos por la autoridad monetaria, mientras que otros tienden a tener menos fondos que los exigidos por la Reserva Federal, por lo cual se origina un mercado interbancario en el que los bancos con excesos de encaje le prestan a los que necesitan cubrir sus posiciones de liquidez. La tasa a la que se realizan dichos préstamos es la tasa de fondos federales. Actualmente dicho porcentaje en los años bajo análisis, 2015 y 2016, es del 1\%.

Si bien la Reserva Federal no controla directamente la tasa de fondos federales, la misma da a conocer luego de las reuniones del FOMC (Comité Federal de Mercado Abierto) una tasa objetivo alrededor de la cual se espera que fluctúe la tasa de fondos federales de mercado. La autoridad monetaria norteamericana realiza operaciones de mercado abierto con el propósito de mantener dicha tasa en el nivel deseado, lo que a su vez induce a otras tasas de la economía a moverse en el mismo sentido. Las operaciones de mercado abierto se materializan a través de la compra o venta de títulos públicos en el mercado por parte de la autoridad monetaria. Cuando la Reserva Federal adquiere títulos públicos inyecta dinero en la economía con lo cual la tasa de fondos federales cae, mientras que cuando vende títulos públicos retira dinero del mercado y la tasa de fondos federales aumenta.
Valor futuro

Uno de los aspectos clave en finanzas es el valor del dinero en el tiempo, en el sentido que siempre un sol oro hoy vale más que un sol oro mañana. Para efectos de poder calcular en forma homogénea los flujos que ocurren en distinto momento en el tiempo, debemos llevar todos estos a un valor presente o a valor futuro.

Valor futuro, es la cantidad de dinero que alcanzará una inversión en alguna fecha futura al ganar intereses a alguna tasa compuesta. El valor que en cualquier caso calculemos depende de los flujos de caja generados por el activo. Es decir, depende de su tamaño, tiempo y riesgo. También, y muy críticamente, el valor depende del costo de oportunidad, ya que para realizar una valoración se deben tener los flujos que ocurren en distintas oportunidades en el tiempo, con riesgos distintos, en una base comparable.

\section{Sobre deterioro de activos fijos}

Veamos a continuación un detenido análisis sobre el tema en la República de Guatemala:

Gallardo, E (2010: 76-106), autor de este trabajo de investigación se ubica en el Municipio de San Lucas, departamento de Sacatepéquez, en la República de Guatemala. Esta referida a una empresa dedicada a la producción y distribución de helados en el mercado nacional y Centro América. La empresa controla el 75\% del consumo del mercado nacional con producción de carácter exclusivo, por esta razón la maquinaria utilizada ha sido fabricada bajo pedidos especiales a solicitud de la dirección, y la supervisión supera los estándares internacionales. Sin embargo, a través de estudios de posicionamiento de mercado, por 
crecimiento de la competencia, registra importante disminución en las proyecciones de ventas, asimismo la recesión de la economía del país ha ocasionado disminución en los volúmenes de venta, ocasionando reducciones significativas en las líneas de producción, al extremo de reducir turnos de operarios y de días laborables para la Planta. Situación que ha provocado que la administración evalúe la posición de la compañía frente al uso de la propiedad, planta y equipo para determinar si existe evidencia sobre si se ha deteriorado su valor, y para tales propósitos se aplica las evaluaciones indicadas en la NIC $\mathrm{N}^{\circ} 36$.

Al 31 de diciembre del 2007, la empresa presentaba en su estado de situación 69 millones de quetzales en activos fijos sobre un total de 93 millones en moneda nacional. Es decir, el 74\% de su inversión total. Y las utilidades antes de impuestos que generaba la empresa en ese mismo periodo, era de $16 \%$ sobre el patrimonio.

En el aspecto de indicadores externos se analizaron: tasas de interés aplicables, evaluación de clientes y proveedores principales, valuación de activos; y no se identificaron cambios importantes. En cuanto a indicadores externos se evaluaron presupuestos planteados a finales de diciembre 2006 con respecto a las cifras obtenidas en el 2007. Técnicos y mecánicos especializados realizaron inspección detallada de los vehículos, congeladores y cuartos fríos, para determinar la existencia de algún daño importante, así mismo se revisaron los planes de ventas y expansiones de clientes.

\section{Tabla 1.}

Distribuidora $G$ (Petén) valor presente de los flujos de efectivo esperados (Cifras expresadas en Quetzales) Fuente: Gallardo E. (2010).

\begin{tabular}{cccc} 
AÑOS & $\begin{array}{c}\text { INGRESOS } \\
\text { PROYECTADOS }\end{array}$ & $\begin{array}{c}\text { FACTOR } \\
\text { DE DESCUENTO } \\
\mathbf{6} \%\end{array}$ & $\begin{array}{c}\text { VALOR PRESENTE } \\
\text { A 2007 }\end{array}$ \\
\hline 2008 & 8177222 & 0,9433962 & 7714360 \\
\hline 2009 & 5724055 & 0,8899964 & 5094388 \\
\hline 2010 & 4293042 & 0,8396193 & 3604521 \\
\hline 2011 & 3434433 & 0,7920937 & 2720393 \\
\hline 2012 & 3221772 & 0,7472582 & 2407496 \\
\hline & $\mathbf{2 4 8 5 0 5 2 4}$ & $\mathbf{2 1 5 4 1 1 5 8}$ \\
\hline & Valor en Libros al 31 de diciembre de 2007 & $\mathbf{2 7 0 2 9 3 8 4}$ \\
\hline & Deterioro & $\mathbf{( 5 4 8 8 2 2 6 )}$
\end{tabular}

La pérdida por deterioro del activo, es la cantidad en que excede el valor en libros del conjunto de la unidad generadora de efectivo, de su importe recuperable, que en este caso se determinó por medio del valor de uso. El efecto de este ajuste se refleja en el resultado del periodo actual. No hay una explicación puntual para haber establecido la tasa de descuento en $6 \%$.

Veamos otro caso de deterioro de activos en la República del Ecuador: Sumba, D. \& Sigua, M. (2012: 15-39). El objetivo de este trabajo consiste en establecer los procedimientos que deben ser aplicados para asegurarse que los activos de la Compañía de Transportes Arcentales Cía. Ltda., (Cuenca, Ecuador), estén contabilizados por un importe que no sea superior a su importe recuperable. Representa un problema cuya solución depende del juicio profesional y la capacidad que la administración de una entidad tenga para el establecimiento de dicha tasa de descuento y de las bases, suposiciones e hipótesis que le dan sustento.

En el caso práctico que presentan los autores consideran como tasa de descuento la tasa de interés activa asociada con el préstamo obtenido por el accionista y que sirvió para la adquisición del vehículo. Bajo las circunstancias del caso, según ellos, esta es la tasa que refleja mejor los riesgos $\mathrm{y}$ beneficios inherentes al funcionamiento del vehículo (10\%). Tasa que sirvió para calcular el VAN. (Valor Actual Neto).

Valor Razonable según la NIC Nº 36. Este tema ha sido analizado con profesionalismo por el Profesor Alejandro Ferrer Quea, desde el año 2010, quién ya advertía, desde esa fecha a la 
actualidad, (mayo 2017), que ya pasaron más de 7 años, que los elementos claves eran la proyección del flujo de efectivo y la identificación de la tasa de descuento.

Según Ferrer, A. (2010: IV-6).

"Para la determinación de este valor razonable se requiere que la gerencia fundamente sus proyecciones de flujos de efectivo ya que éstos resultan importes claves en el cálculo, también lo es la identificación de la tasa de descuento".

Analicemos el caso práctico de Ferrer, A. (2013: IV-13), nos ha llamado la atención por la tasa de descuento que se utiliza: $15 \%$. Lo desarrollaremos in-extenso, por ser un tema que en el mediano plazo se dará en el Perú: por cambio de tecnología.

\section{Desvalorización de Planta Industrial}

Enunciado.

La empresa Concentrados Eléctricos construyó una planta eléctrica a base de petróleo que ha estado usando por varios años, siendo su valor neto en libros al 31 de diciembre del 2010 de $\mathrm{S} / .800,000$.
Debido al alza del precio del petróleo, la compañía ha comprado una nueva planta eléctrica a base de gas natural.

En los próximos años, solo va a utilizar la planta a base de petróleo cuando exceda la capacidad de la nueva

planta y por consiguiente, su flujo de efectivo va a disminuir.

La empresa ha dispuesto efectuar un estudio de desvalorización de la mencionada planta. ¿Cómo determinar la desvalorización de la planta eléctrica?

\section{Planteamiento del Problema.}

a) Determinar el valor razonable (valor de venta).

b) Establecer el valor de uso:

Definir el flujo de efectivo

Identificar la Tasa de Interés para calcular el factor de descuento.

c) Estimación de la desvalorización.

d) Registro contable.

2. Desarrollo del Caso

a) Determinar el valor razonable (valor de venta)

Por tasación de Perito Independiente $=$ Valor razonable $\mathrm{S} / 700,000$

Gastos de desmontaje

b) Establecer el valor de uso

Tasa de interés, según condiciones actual del mercado =

$15 \%$

Factor de descuento $=\mathrm{I} /(1+\mathrm{i}) \mathrm{n}$

F. D. = Factor de Descuento $=$ ? $\mathrm{i}=$ tasa de interés $=15 \%$ anual $\mathrm{n}=$ número de periodos $=8$ años
Proyección de flujo de efectivo para los próximos 8 años que le queda de vida útil a la planta. (Ver Tabla 2)

Tabla 2 .

Empresa concentrados eléctricos flujos futuros de efectivo descontado (valor expresado en miles de soles) Fuente: Ferrer, A. (2013, IV-11) Revisando las NIIF: NIC N. 36 Deterioro del Valor de los Activos. Revista Actualidad Empresarial” No. 277. Segunda Quincena de abril 2013. Lima. Perú.

\begin{tabular}{cccc} 
AÑOS & $\begin{array}{c}\text { INGRESOS } \\
\text { PROYECTADOS }\end{array}$ & $\begin{array}{c}\text { FACTOR DE } \\
\text { DESCUENTO 15\% }\end{array}$ & $\begin{array}{c}\text { FLUJO DE EFECTIVO } \\
\text { DESCONTADO }\end{array}$ \\
2011 & 180 & 0,8695652 & 156,52 \\
\hline 2012 & 150 & 0,7561437 & 713,42 \\
\hline 2013 & 120 & 0,6575162 & 57,90 \\
\hline 2014 & 100 & 0,5717532 & 42,26 \\
\hline 2015 & 85 & 0,4971767 & 25,94 \\
\hline 2016 & 60 & 0,4323276 & 15,79 \\
\hline 2017 & 42 & 0,3759370 & 13,08
\end{tabular}


1. Estimación de la desvalorización.

Valor Razonable (Venta)

S/. 600000

Valor de Uso (Ver Tabla 4).

S/. 503090

Se elige el valor razonable por ser el mayor. Luego se confronta con el valor en libros:

Valor en libros

S/. 800000

Valor Razonable (Venta)

Monto a Desvalorizar

2. Registro Contable.

68 VALUACIÓN Y DETERIORO DE ACTIVOS Y PROVISIONES

200000

685 deterioro del valor de los activos

36 DESVALORIZACIÓN DE ACTIVO INMOVILIZ.

200000

3632 instalaciones

$\mathrm{x} /$ Provisión del gasto.

94 GASTOS DE ADMINISTRACIÓN

200000

79 CARGAS IMPUTABLES A CUENTAS DE COSTOS

200000

$\mathrm{x} /$ Destino del gasto

En resumen, notamos aquí, de tres trabajos desarrollados a nivel de América Latina, el siguiente resultado en cuanto a tasas de interés aplicado a flujos futuros: Ecuador 10\%. Guatemala $6 \%$. Perú $15 \%$. Son diferentes realidades, pero un solo problema. Es decir, buscamos la unidad en la diver- sidad. Si se aplicara la tasa de interés neutral se tendrían las siguientes tasas en base a los años 2015-2016:

Tabla 3.

Comparación de tasas a valor de mercado con tasas neutrales (Expresado en porcentaje)

Fuente: Evaluación propia. Tasas tomadas de las tablas desarrolladas por el autor.

\begin{tabular}{cccc}
\multirow{2}{*}{ PAÍSES } & TASAS A VALOR DE & \multicolumn{2}{c}{ TASAS NEUTRALES } \\
\cline { 3 - 4 } & MERCADO & $2015(1)$ & $2016(2)$ \\
\hline Ecuador & $10 \%$ & $17,53 \%$ & $9,59 \%$ \\
\hline Guatemala & $6 \%$ & $3,60(3)$ & $3,30 \%(3)$ \\
\hline Perú & $15 \%$ & 7,08 & $6,45 \%$ \\
\hline
\end{tabular}

(1) Cuadro 8. Tasa de Interés Global Neutral de Países Emergentes año 2015.

(2) Cuadro 11. Tasa de Interés Global Neutral de Países Emergentes año 2016.

(3) No incluye Riesgo País.

Este cuadro nos muestra que las tasas neutrales son más altas que las tasas a valor de mercado, porque no incluyen el factor inflación. Pero en la medida que se recupera la economía del país, tienden a la baja. Se premia al país cuando usa eficientemente los instrumentos macroeconómicos y se "castiga" cuando ocurre lo contrario. Perú, por manejar adecuadamente su economía tiene tasas súper competitivas en el mercado global.

\section{MATERIAL Y MÉTODOS}

Se utilizó una metodología no experimental de caso, fundamentalmente cuantitativa, exploratoria-descriptiva, puesto que los problemas y cuestiones 
planteados en los objetivos, son aspectos dinámicos difíciles de estudiar mediante una posición estrictamente cualitativa, porque detallamos las características del proceso económico-financiero en aplicación de la tasa de interés a efectos de cumplir con la aplicación de las Normas Internacionales de Información Financiera.

Unidad de análisis, son todas las empresas, públicas o privadas, que aplican la NIC 19, NIC 36, NIC 37 y NIIF 13. Y la población de estudio está representada por todas las empresas de América Latina y el Caribe, que tienen la necesidad de aplicar una tasa de interés neutral para calcular el valor futuro de sus flujos de caja y cuya única variable es el porcentaje de la tasa de interés para determinar su factor de descuento. En cuanto a la muestra, es no probabilístico por conveniencia. Porque se basa en dos flujos de caja que se muestran en las Tablas 4 y 5 . Mientras que en la primera las tasas van de menos a más; en la segunda de más a menos.
La principal técnica que se manejó como recolección de información en este estudio fue el análisis económico-financiero, es decir, una aplicación de simulación matemática.

\section{RESULTADOS}

El análisis cuantitativo nos muestra que, a mayor tasa de interés, (pasamos de $6 \%$ a $12 \%$ ), el deterioro aumenta, por tanto, la empresa tiene mayor crédito fiscal, y pagará menos impuestos a la renta, afectando los intereses del Estado. Pero si aplicamos las tasas neutrales que figuran en las Tablas $\mathrm{N}^{\circ} .8,9,10,11$ y 12 , se observan los siguientes resultados:

En los países de economía avanzada (ver Tablas 7 y 10), sus tasas fluctúan en el rango de $4,1 \%$ a $0,300 \%$ en el año 2015. Corresponden a los países de Hong Kong y Suiza por tener la más alta y la más baja inflación respectivamente. Es decir, a mayor eficiencia en el manejo de los instrumentos de política macroeconómica correspon- de tasas más bajas de interés neutral. En el año 2016, se confirma esta tendencia (Ver Tabla 11). Los rangos de las tasas neutrales fluctúan entre $4,7 \%$ a 0,6\%. Que corresponde los países de Noruega y Singapur, respectivamente, por tener la más alta y la más baja inflación.

En los países emergentes (ver Tablas 7 y 10), sus tasas fluctúan en el rango de $149,488 \%$ a $0,208 \%$ en el año 2015 . Corresponden a los países de Venezuela y Tailandia por tener la más alta y la más baja inflación respectivamente. Es decir, a mayor deficiencia en el manejo de los instrumentos de política macroeconómica corresponde tasas más altas de interés neutral. En el año 2016, se confirma esta tendencia (Ver Tabla 10). Los rangos de las tasas neutrales fluctúan entre $277,810 \%$ a 0,6\%. Que corresponde los países de Venezuela y Polonia, respectivamente, por tener la más alta y la más baja inflación. Venezuela incrementa su tasa porque la crisis económica continúa deteriorándose más.

Tabla 4.

Distribuidora $G$ (Petén). valor presente de los flujos de efectivo esperados (cifras expresadas en quetzales)

Fuente: Propia. Modelo de simulación matemática.

\begin{tabular}{cccccc}
\multirow{2}{*}{ AÑOS } & INGRESOS PROYECTADOS & \multicolumn{2}{c}{ FACTOR DE DESCUENTO } & \multicolumn{2}{c}{ FACTOR DE DESCUENTO } \\
& & $\mathbf{6} \%$ & VALOR PRESENTE & $\mathbf{1 2 \%}$ & VALOR PRESENTE \\
\hline 2008 & 8177222 & 0,9433962 & 7714360 & 0,8928571 & 7301091 \\
\hline 2009 & 57254055 & 0,8899964 & 5094389 & 0,7971939 & 4563182 \\
\hline 2010 & 4293042 & 0,8396193 & 3604520 & 0,7117802 & 3055702 \\
\hline 2011 & 3434433 & 0,7920937 & 2720393 & 0,6355181 & 2182644 \\
\hline 2012 & 3221772 & 0,7472582 & 2407496 & 0,5674269 & 1828120 \\
\hline \multicolumn{3}{c}{ Valor en libros al 31 de diciembre 2007 } & 21541158 & & 18930739 \\
\hline & Deterioro & 27029384 & & $(54029384$ \\
\hline
\end{tabular}

Veamos a continuación la siguiente simulación matemática:

En este caso, al caer la tasa de interés de $15 \%$ a 7,5\% anual, el valor de deterioro también se reduce. 
Tabla 5.

Empresa concentrados eléctricos flujos futuros de efectivo descontado (valor expresado en miles de soles) Fuente: Propia. Modelo de simulación matemática.

\begin{tabular}{cccccc}
\multirow{2}{*}{ AÑOS } & INGRESOS PROYECTADOS & \multicolumn{2}{c}{ FACTOR DE DESCUENTO } & \multicolumn{2}{c}{ FACTOR DE DESCUENTO } \\
& & $\mathbf{1 5 \%}$ & VALOR PRESENTE & $\mathbf{7 , 5} \%$ & VALOR PRESENTE \\
\hline 2011 & 180 & 0,8695652 & 156,52 & 0,9302326 & 167,44 \\
\hline 2012 & 150 & 0,7561437 & 113,42 & 0,8653326 & 129,80 \\
\hline 2013 & 120 & 0,6575162 & 78,90 & 0,8049606 & 96,60 \\
\hline 2014 & 100 & 0,5717532 & 57,18 & 0,7488005 & 74,88 \\
\hline 2015 & 85 & 0,4971767 & 42,26 & 0,6965586 & 59,21 \\
\hline 2016 & 60 & 0,4323276 & 25,94 & 0,6479615 & 38,88 \\
\hline 2017 & 42 & 0,3759370 & 15,79 & 0,6027549 & 25,32 \\
\hline 2018 & 40 & 0,3269018 & 13,08 & 0,5607022 & 22,43 \\
TOTAL & 777 & & $\mathbf{5 0 3 , 0 9}$ & & $\mathbf{6 1 4 , 5 6}$ \\
& Valor en Libros & & $\mathbf{8 0 0 , 0 0}$ & & $\mathbf{8 0 0 , 0 0}$ \\
& Deterioro & & $\mathbf{( 2 9 6 , 9 1 )}$ & & $(\mathbf{2 1 4 , 5 6 )}$
\end{tabular}

A la luz de la información precedente, sustentada en términos cuantitativos, se deduce que la tasa de interés está en relación directa con el valor del deterioro. A mayor tasa de interés, más deterioro. Por tanto, es una variable que debe ser controlada. No puede dejarse al libre albedrío de los valuadores y/o los empresarios del mundo. Razón más que suficiente para fiscalizarla.

“QQué hubiera pasado si China esperaba hasta que la FED decidiera una suba de tasas para resolver, recién entonces, la devaluación del yuan renminbí?" se preguntaban Barbera, J \& Gutiérrez, A \& Siaba, J. (2015: 47). Concluyen estos economistas argentinos que "las decisiones económicas son partidas simultáneas que se juegan en un único tablero", el mundo. La coordinación es fundamental para ordenar el tráfico de este mundo global que nos tocó vivir.

Nuestra Propuesta: Tasa de Interés Latinoamericana Neutral.

Los componentes de la tasa de interés neutra que proponemos son los siguientes:

a) Tasas de interés de fondos federales de Estados Unidos de Norteamérica. Es el precio que se paga por el dinero en el mercado interbancario norteamericano por préstamos a un día (overnight). Es una tasa de interés libre ya que no está regulada directamente por la Reserva Federal de Estados Unidos de Norte América.

b) La inflación o devaluación, es manejado por los Bancos Centrales del mundo, en nuestro país, el Perú, es el Banco Central de Reserva.

c) El riesgo país, representado por el Emerging Market Bond Index (EMBI), indicador calculado por el banco JP Morgan Chase. Este factor, es una estimación del costo de endeudamiento y de la vulnerabilidad que el mercado percibe en una economía.

d) Gastos administrativos, que es el costo que cobran los bancos para gerenciar el dinero.

e) La sumatoria de estos elementos en cualquier parte del mundo, sería:

$\mathrm{A}+\mathrm{b}+\mathrm{c}+\mathrm{d}=$ Tasa de interés global

Tabla 6.

Estructura de una tasa de interés latinoamericana neutral para aplicación de las normas internacionales de información financiera Fuente: Propia. Tabla propuesta por el Autor de la investigación.

\begin{tabular}{llllll}
\multicolumn{1}{c}{ Países } & Tasa Fed & Inflación (devaluación) & Riesgo País & Gastos Administrativos & TOTAL \\
Economía Avanzada & & & \\
\hline Emergentes & \\
\hline No Emergentes
\end{tabular}


Tabla 7.

Tasa de interés global neutral para países de economía avanzada. Diciembre 2015. (Expresado en tanto por ciento) Fuente: MSCI.COM; Fondo Monetario Internacional; Fed; http://es.global-rates.com/estadísticas-económicas/inflación/2015. aspx.

\begin{tabular}{|c|c|c|c|c|c|}
\hline Países & Tasa Fed & Inflación (devaluación) & $\begin{array}{l}\text { Riesgo } \\
\text { País }\end{array}$ & Gastos Administrativos & TOTAL \\
\hline Alemania & 1,000 & 0,281 & 0 & 0,100 & 1,381 \\
\hline Australia & 1,000 & 1,700 & 0 & 0,100 & 2,800 \\
\hline Austria & 1,000 & 1,019 & 0 & 0,100 & 2,119 \\
\hline Bélgica & 1,000 & 1,500 & 0 & 0,100 & 2,600 \\
\hline Canadá & 1,000 & 1,606 & 0 & 0,100 & 2,706 \\
\hline Dinamarca & 1,000 & 0,402 & 0 & 0,100 & 1,502 \\
\hline España & 1,000 & 0,017 & 0 & 0,100 & 1,117 \\
\hline $\begin{array}{l}\text { Estados Unidos de } \\
\text { N.A. }\end{array}$ & 1,000 & 0,730 & 0 & 0,100 & 1,830 \\
\hline Finlandia & 1,000 & $-0,239$ & 0 & 0,100 & 0,861 \\
\hline Francia & 1,000 & 0,180 & 0 & 0,100 & 1,280 \\
\hline Gran Bretaña & 1,000 & 0,200 & 0 & 0,100 & 1,300 \\
\hline Holanda & 1,000 & 0,700 & 0 & 0,100 & 1,800 \\
\hline Hong-Kong & 1,000 & 3,000 & 0 & 0,100 & 4,100 \\
\hline Irlanda & 1,000 & 0,100 & 0 & 0,100 & 1,200 \\
\hline Islandia & 1,000 & 2,029 & 0 & 0,100 & 3,129 \\
\hline Israel & 1,000 & $-0,965$ & 0 & 0,100 & 0,135 \\
\hline Italia & 1,000 & 0,093 & 0 & 0,100 & 1,193 \\
\hline Japón & 1,000 & 0,100 & 0 & 0,100 & 1,200 \\
\hline Luxemburgo & 1,000 & 1,093 & 0 & 0,100 & 2,193 \\
\hline Noruega & 1,000 & 2,333 & 0 & 0,100 & 3,433 \\
\hline Nueva Zelanda & 1,000 & 0,300 & 0 & 0,100 & 1,400 \\
\hline Portugal & 1,000 & 0,397 & 0 & 0,100 & 1,497 \\
\hline Singapur & 1,000 & $-0,500$ & 0 & 0,100 & 0,600 \\
\hline Suecia & 1,000 & 0,051 & 0 & 0,100 & 1,151 \\
\hline Suiza & 1,000 & $-1,308$ & 0 & 0,100 & $-0,208$ \\
\hline
\end{tabular}

Tabla 8.

Tasa de interés global neutral para países emergentes. Diciembre 2015.(Expresado en tanto por ciento) Fuente: MSCI.COM.Fed.CEPAL.BCRP. FMI.JP Morgan.

(1)Asumido. (2) s/i= sin información

\begin{tabular}{lccccc}
\multicolumn{1}{c}{ Países } & Tasa Fed & Inflación (devaluación) & $\begin{array}{c}\text { Riesgo } \\
\text { País }\end{array}$ & $\begin{array}{c}\text { Gastos Administrativos } \\
(\mathbf{1})\end{array}$ & TOTAL \\
Argentina & 1,000 & 26,500 & 4,780 & 0,200 & 32,480 \\
\hline Bolivia & 1,000 & 4,100 & 2,500 & 0,200 & 7,800 \\
\hline
\end{tabular}

Continúa en la siguiente página 


\begin{tabular}{|c|c|c|c|c|c|}
\hline Brasil & 1,000 & 9,000 & 5,120 & 0,200 & 15,320 \\
\hline Colombia & 1,000 & 5,000 & 3,140 & 0,200 & 9,340 \\
\hline Corea del Sur & 1,000 & 1,132 & s/i (2) & 0,200 & 2,332 \\
\hline China & 1,000 & 1,616 & $\mathrm{~s} / \mathrm{i}$ & 0,200 & 2,816 \\
\hline Chile & 1,000 & 4,300 & 2,500 & 0,200 & 8,000 \\
\hline Ecuador & 1,000 & 4,000 & 12,330 & 0,200 & 17,530 \\
\hline El Salvador & 1,000 & $-0,700$ & 6,340 & 0,200 & 6,840 \\
\hline Eslovaquia & 1,000 & $-0,489$ & $\mathrm{~s} / \mathrm{i}$ & 0,200 & 0,711 \\
\hline Grecia & 1,000 & $-0,167$ & $\mathrm{~s} / \mathrm{i}$ & 0,200 & 1,033 \\
\hline Guatemala & 1,000 & 2,400 & $\mathrm{~s} / \mathrm{i}$ & 0.200 & 3,600 \\
\hline Hungría & 1,000 & 0,899 & $\mathrm{~s} / \mathrm{i}$ & 0,200 & 2,099 \\
\hline India & 1,000 & 4,900 & $\mathrm{~s} / \mathrm{i}$ & 0,200 & 6,100 \\
\hline Indonesia & 1,000 & 6,400 & $\mathrm{~s} / \mathrm{i}$ & 0,200 & 7,600 \\
\hline Jamaica & 1,000 & 4,700 & 4,690 & 0,200 & 10,590 \\
\hline Malasia & 1,000 & 2,100 & $\mathrm{~s} / \mathrm{i}$ & 0,200 & 3,300 \\
\hline México & 1,000 & 2,131 & 3,110 & 0,200 & 6,441 \\
\hline Panamá & 1,000 & 0,100 & 2,180 & 0,200 & 3,480 \\
\hline Paraguay & 1,000 & 2,900 & 3,380 & 0,200 & 7,480 \\
\hline Perú & 1,000 & 3,500 & 2,380 & 0,200 & 7,080 \\
\hline Polonia & 1,000 & $-0,650$ & $\mathrm{~s} / \mathrm{i}$ & 0,200 & 0,550 \\
\hline Rep. Checa & 1,000 & 0,300 & $\mathrm{~s} / \mathrm{i}$ & 0,200 & 1,500 \\
\hline Rep. Dominicana & 1,000 & 0,800 & 4,210 & 0,200 & 6,210 \\
\hline Rusia & 1,000 & 15,500 & $s / i$ & 0,200 & 16,700 \\
\hline Sudáfrica & 1,000 & 4,600 & $\mathrm{~s} / \mathrm{i}$ & 0,200 & 5,800 \\
\hline Tailandia & 1,000 & $-0,900$ & $\mathrm{~s} / \mathrm{i}$ & 0,200 & 0,300 \\
\hline Taiwán & 1,000 & $-0,300$ & $\mathrm{~s} / \mathrm{i}$ & 0,200 & 0,900 \\
\hline Turquía & 1,000 & 8,770 & s/i & 0,200 & 9,970 \\
\hline Uruguay & 1,000 & 8,700 & 2,800 & 0,200 & 12,700 \\
\hline Venezuela & 1,000 & 121,700 & 26,580 & 0,200 & 149,488 \\
\hline
\end{tabular}

Tabla 9.

Tasas de interés global neutrales países no emergentes. Diciembre 2015. (Expresado en tanto por ciento) Fuente: MSCI.COM. Fed. FMI. (1) s/i=sin información. (2) Asumido.

\begin{tabular}{lccccc}
\multicolumn{1}{c}{ Países } & Tasa Fed & Inflación (devaluación) & $\begin{array}{c}\text { Riesgo } \\
\text { País (1) }\end{array}$ & $\begin{array}{c}\text { Gastos Administrativos } \\
\text { (2) }\end{array}$ & TOTAL \\
Bangladesh & 1,000 & 6,400 & s/i & 0,200 & 7,600 \\
\hline Bielorrusia & 1,000 & 13,500 & s/i & 0,200 & 14,700 \\
\hline $\begin{array}{l}\text { Bosnia y Herzego- } \\
\text { vina }\end{array}$ & 1,000 & $-1,000$ & s/i & 0,200 & 0,200 \\
\hline
\end{tabular}

Continúa en la siguiente página 


\begin{tabular}{|c|c|c|c|c|c|}
\hline Botsuana & 1,000 & 3,000 & s/i & 0,200 & 4,200 \\
\hline Bulgaria & 1,000 & $-1,100$ & $\mathrm{~s} / \mathrm{i}$ & 0,200 & 0,100 \\
\hline Croacia & 1,000 & $-0,500$ & $\mathrm{~s} / \mathrm{i}$ & 0,200 & 0,700 \\
\hline Eslovenia & 1,000 & $-0,500$ & $\mathrm{~s} / \mathrm{i}$ & 0,200 & 0,700 \\
\hline Estonia & 1,000 & 0,100 & $\mathrm{~s} / \mathrm{i}$ & 0,200 & 1,300 \\
\hline Ghana & 1,000 & 17,200 & $\mathrm{~s} / \mathrm{i}$ & 0,200 & 18,400 \\
\hline Kazajistán & 1,000 & 6,500 & $\mathrm{~s} / \mathrm{i}$ & 0,200 & 7,700 \\
\hline Kenya & 1,000 & 6,600 & $\mathrm{~s} / \mathrm{i}$ & 0,200 & 7,800 \\
\hline Liberia & 1,000 & 7,700 & $\mathrm{~s} / \mathrm{i}$ & 0,200 & 8,900 \\
\hline Lituania & 1,000 & $-0,700$ & $\mathrm{~s} / \mathrm{i}$ & 0,200 & 0,500 \\
\hline Mongolia & 1,000 & 5,900 & $\mathrm{~s} / \mathrm{i}$ & 0,200 & 7,100 \\
\hline Nigeria & 1,000 & 9,000 & $\mathrm{~s} / \mathrm{i}$ & 0,200 & 10,200 \\
\hline Rumanía & 1,000 & $-0,600$ & $\mathrm{~s} / \mathrm{i}$ & 0,200 & 0,600 \\
\hline Sao Tomé & 1,000 & 5,300 & $\mathrm{~s} / \mathrm{i}$ & 0,200 & 6,500 \\
\hline Serbia & 1,000 & 1,400 & $\mathrm{~s} / \mathrm{i}$ & 0,200 & 2,600 \\
\hline Tanzania & 1,000 & 5,600 & $\mathrm{~s} / \mathrm{i}$ & 0,200 & 6,800 \\
\hline Trinidad y Tobago & 1,000 & 4,700 & $\mathrm{~s} / \mathrm{i}$ & 0,200 & 5,900 \\
\hline Turkmenistán & 1,000 & 5,500 & $\mathrm{~s} / \mathrm{i}$ & 0,200 & 6,700 \\
\hline Ucrania & 1,000 & 48,700 & $\mathrm{~s} / \mathrm{i}$ & 0,200 & 49,900 \\
\hline Vietnam & 1,000 & 0,600 & $\mathrm{~s} / \mathrm{i}$ & 0,200 & 1,800 \\
\hline Zimbabwe & 1,000 & $-2,400$ & $\mathrm{~s} / \mathrm{i}$ & 0,200 & $-1,200$ \\
\hline
\end{tabular}

Tabla 10.

Tasas de interés global neutrales para países de economía avanzada. Diciembre 2016. (Expresado en tanto por ciento) Fuente: MSCI.COM; Fondo Monetario Internacional; Fed; http://es.global-rates.com/estadísticas-económicas/inflación/2015. aspx

\begin{tabular}{lccccc}
\multicolumn{1}{c}{ Países } & Tasa Fed & Inflación (devaluación) & $\begin{array}{c}\text { Riesgo } \\
\text { País }\end{array}$ & Gastos Administrativos & TOTAL \\
Alemania & 1,000 & 0,400 & 0 & 0,100 & 1.500 \\
\hline Australia & 1,000 & 1,300 & 0 & 0,100 & 2,400 \\
\hline Austria & 1,000 & 1,000 & 0 & 0,100 & 2,100 \\
\hline Bélgica & 1,000 & 1,800 & 0 & 0,100 & 2,900 \\
\hline Canadá & 1,000 & 1,400 & 0 & 0,100 & 2,500 \\
\hline Dinamarca & 1,000 & 0,300 & 0 & 0,100 & 1,400 \\
\hline España & 1,000 & $-0,200$ & 0 & 0,100 & 0,900 \\
\hline Estado Unidos de & 1,000 & 1,300 & 0 & 0,100 & 2,400 \\
\hline N.A. & 1,000 & 0,300 & 0 & 0,100 & 2,400 \\
\hline Francia & 1,000 & 0,400 & 0 & 0,100 & 1,500 \\
\hline Finlandia & & & & \\
\hline
\end{tabular}




\begin{tabular}{llllll}
\hline Gran Bretaña & 1,000 & 0,600 & 0 & 0,100 & 1,700 \\
\hline Holanda & 1,000 & 0,100 & 0 & 0,100 & 1,200 \\
\hline Hong-Kong & 1,000 & 2,600 & 0 & 0,100 & 2,700 \\
\hline Irlanda & 1,000 & $-0,200$ & 0 & 0,100 & 0,900 \\
\hline Islandia & 1,000 & 1,700 & 0 & 0,100 & 2,800 \\
\hline Israel & 1,000 & $-0,500$ & 0 & 0,100 & 0,600 \\
\hline Italia & 1,000 & $-0,100$ & 0 & 0,100 & 1,000 \\
\hline Japón & 1,000 & 0,100 & 0 & 0,100 & 1,200 \\
\hline Luxemburgo & 1,000 & 0,100 & 0 & 0,100 & 1,200 \\
\hline Noruega & 1,000 & 3,600 & 0 & 0,100 & 4,700 \\
\hline Nueva Zelanda & 1,000 & 0,600 & 0 & 0,100 & 1,700 \\
\hline Portugal & 1,000 & 0,600 & 0 & 0,100 & 1,700 \\
\hline Singapur & 1,000 & $-0,500$ & 0 & 0,100 & 0,600 \\
\hline Suecia & 1,000 & 1,100 & 0 & 0,100 & 2,200 \\
\hline Suiza & 1,000 & $-0,400$ & 0 & 0,100 & 0,700 \\
\hline
\end{tabular}

Tabla 11.

Tasas de interés global neutrales para países emergentes. Diciembre 2016. (Expresado en tanto por ciento) Fuente: MSCI.COM. Fed. CEPAL. BCRP. FMI. JP Morgan. (1) Asumido. (2) s/i=sin información

\begin{tabular}{|c|c|c|c|c|c|}
\hline Países & Tasa Fed & Inflación (devaluación) & $\begin{array}{l}\text { Riesgo } \\
\text { País }\end{array}$ & $\begin{array}{c}\text { Gastos Administrativos } \\
\text { (2) }\end{array}$ & TOTAL \\
\hline Argentina & 1,000 & $\mathrm{~s} / \mathrm{i}(1)$ & 4,940 & 0,200 & 6,140 \\
\hline Bolivia & 1,000 & 3,600 & $\mathrm{~s} / \mathrm{i}$ & 0,200 & 4,800 \\
\hline Brasil & 1,000 & 8,700 & 3,330 & 0,200 & 13,230 \\
\hline Colombia & 1,000 & 7,500 & 2,280 & 0,200 & 10,980 \\
\hline Corea del Sur & 1,000 & 1,000 & $\mathrm{~s} / \mathrm{i}$ & 0,200 & 2,200 \\
\hline China & 1,000 & 2,000 & $\mathrm{~s} / \mathrm{i}$ & 0,200 & 3,200 \\
\hline Chile & 1,000 & 3,800 & 1,620 & 0,200 & 6,620 \\
\hline Ecuador & 1,000 & 1,700 & 6,690 & 0,200 & 9,590 \\
\hline El Salvador & 1,000 & 2,100 & $\mathrm{~s} / \mathrm{i}$ & 0,200 & 3,300 \\
\hline Eslovaquia & 1,000 & $-0,500$ & $\mathrm{~s} / \mathrm{i}$ & 0,200 & 0,700 \\
\hline Grecia & 1,000 & 0,000 & $\mathrm{~s} / \mathrm{i}$ & 0,200 & 1,200 \\
\hline Guatemala & 1,000 & 2,100 & $\mathrm{~s} / \mathrm{i}$ & 0,200 & 3,300 \\
\hline Hungría & 1,000 & 0,400 & $\mathrm{~s} / \mathrm{i}$ & 0,200 & 1,600 \\
\hline India & 1,000 & 4,900 & $\mathrm{~s} / \mathrm{i}$ & 0,200 & 6,100 \\
\hline Indonesia & 1,000 & 3,500 & $\mathrm{~s} / \mathrm{i}$ & 0,200 & 4,700 \\
\hline Jamaica & 1,000 & 2,800 & s/i & 0,200 & 4,000 \\
\hline Malasia & 1,000 & 2,100 & $\mathrm{~s} / \mathrm{i}$ & 0,200 & 3,300 \\
\hline México & 1,000 & 2,800 & 3,020 & 0,200 & 7,020 \\
\hline
\end{tabular}

Continúa en la siguiente página 


\begin{tabular}{|c|c|c|c|c|c|}
\hline Panamá & 1,000 & 2,100 & $s / i$ & 0,200 & 3,300 \\
\hline Paraguay & 1,000 & 4,100 & $\mathrm{~s} / \mathrm{i}$ & 0,200 & 5,300 \\
\hline Perú & 1,000 & 3,600 & 1,650 & 0,200 & 6,450 \\
\hline Polonia & 1,000 & $-0,600$ & $\mathrm{~s} / \mathrm{i}$ & 0,200 & 0,600 \\
\hline Rep. Checa & 1,000 & 0,700 & s/i & 0,200 & 1,900 \\
\hline Rep. Dominicana & 1,000 & 2,800 & $s / i$ & 0,200 & 4,000 \\
\hline Rusia & 1,000 & 7,000 & s/i & 0,200 & 1,900 \\
\hline Sudáfrica & 1,000 & 7,066 & $s / i$ & 0,200 & 8,266 \\
\hline Tailandia & 1,000 & 0,200 & s/i & 0,200 & 1,400 \\
\hline Taiwán & 1,000 & 1,400 & $\mathrm{~s} / \mathrm{i}$ & 0,200 & 2,600 \\
\hline Turquía & 1,000 & 7,800 & $\mathrm{~s} / \mathrm{i}$ & 0,200 & 8,000 \\
\hline Uruguay & 1,000 & 9,600 & $\mathrm{~s} / \mathrm{i}$ & 0,200 & 10,800 \\
\hline Venezuela & 1,000 & 254,900 & 21,710 & 0,200 & 277,810 \\
\hline
\end{tabular}

Tabla 12 .

Tasa de interés global neutrales países no emergentes. Diciembre 2016. (Expresado en tanto por ciento). Fuente: MSCI.COM.Fed. FMI. (1) s/i = sin información. (2) Asumido.

\begin{tabular}{|c|c|c|c|c|c|}
\hline Países & Tasa Fed & Inflación (devaluación) & $\begin{array}{l}\text { Riesgo } \\
\text { País (1) }\end{array}$ & $\begin{array}{c}\text { Gastos Administrativos } \\
\text { (2) }\end{array}$ & TOTAL \\
\hline Bangladesh & 1,000 & 2,400 & $\mathrm{~s} / \mathrm{i}$ & 0,200 & 3,600 \\
\hline Bielorrusia & 1,000 & 11,800 & $\mathrm{~s} / \mathrm{i}$ & 0,200 & 13,000 \\
\hline $\begin{array}{l}\text { Bosnia y Herzego- } \\
\text { vina }\end{array}$ & 1,000 & 3,200 & $\mathrm{~s} / \mathrm{i}$ & 0,200 & 4,400 \\
\hline Botsuana & 1,000 & 6,800 & $\mathrm{~s} / \mathrm{i}$ & 0,200 & 8,000 \\
\hline Bulgaria & 1,000 & $-1,300$ & $\mathrm{~s} / \mathrm{i}$ & 0,200 & $-0,100$ \\
\hline Croacia & 1,000 & $-1,100$ & $\mathrm{~s} / \mathrm{i}$ & 0,200 & 0,100 \\
\hline Eslovenia & 1,000 & $-0,100$ & $\mathrm{~s} / \mathrm{i}$ & 0,200 & 1,100 \\
\hline Estonia & 1,000 & 0,800 & $\mathrm{~s} / \mathrm{i}$ & 0,200 & 2,000 \\
\hline Ghana & 1,000 & 17,500 & $\mathrm{~s} / \mathrm{i}$ & 0,200 & 18,700 \\
\hline Kazajistán & 1,000 & 14,600 & $\mathrm{~s} / \mathrm{i}$ & 0,200 & 15,800 \\
\hline Kenya & 1,000 & 6,300 & $\mathrm{~s} / \mathrm{i}$ & 0,200 & 7,500 \\
\hline Liberia & 1,000 & 7,000 & $\mathrm{~s} / \mathrm{i}$ & 0,200 & 8,200 \\
\hline Lituania & 1,000 & 0,700 & $\mathrm{~s} / \mathrm{i}$ & 0,200 & 1,900 \\
\hline Mongolia & 1,000 & 5,600 & $\mathrm{~s} / \mathrm{i}$ & 0,200 & 6,800 \\
\hline Nigeria & 1,000 & 15,700 & $\mathrm{~s} / \mathrm{i}$ & 0,200 & 16,900 \\
\hline Rumanía & 1,000 & $-1,600$ & $\mathrm{~s} / \mathrm{i}$ & 0,200 & $-0,400$ \\
\hline Sao Tomé & 1,000 & 7,000 & $\mathrm{~s} / \mathrm{i}$ & 0,200 & 7,800 \\
\hline Serbia & 1,000 & 1,100 & $\mathrm{~s} / \mathrm{i}$ & 0,200 & 2,300 \\
\hline Tanzania & 1,000 & 5,200 & $\mathrm{~s} / \mathrm{i}$ & 0,200 & 6,400 \\
\hline
\end{tabular}

Continúa en la siguiente página 


\begin{tabular}{llllll}
\hline Trinidad y Tobago & 1,000 & 2,800 & s/i & 0,200 & 4,000 \\
\hline Turkmenistán & 1,000 & 3,500 & s/i & 0,200 & 4,700 \\
\hline Ucrania & 1,000 & 13,900 & s/i & 0,200 & 15,100 \\
\hline Vietnam & 1,000 & 2,700 & s/i & 0,200 & 3,900 \\
\hline Zimbabwe & 1,000 & 7,000 & s/i & 0,200 & 8,200 \\
\hline
\end{tabular}

\section{El tiempo}

Se propone a los Bancos Centrales de Reserva de América Latina y el Caribe, normar, no controlar, la tasa de interés global neutra, en forma trimestral.

\section{Principio de Transparencia}

- Estaría bajo la supervisión de las siguientes instituciones mundiales:

- Reserva Federal de Estados Unidos de Norteamérica (FED).

- Bancos Centrales de cada país de América Latina.

- El Banco de inversiónJ.P. Morgan Chase.

- Los Contadores Públicos de la región.

\section{DISCUSIÓN}

Ahora es el momento para normar las tasas de interés real neutrales por parte de los Bancos Centrales de Reserva de América Latina y el Caribe, en periodos trimestrales utilizando las dos lenguas oficiales de la Organización de las Naciones Unidas (ONU): español e inglés. Aplicando criterios mundiales ya conocidos y emitidos por organizaciones internacionales de reconocido prestigio, tales como: Reserva Federal de Estados Unidos de Norte América, Bancos Centrales de Reserva de cada país latinoamericano y el Banco de inversiones J.P. Morgan Chase de New York. Así, se anticiparía a la futura crisis que se prevé en el mediano plazo de una gran recesión mundial por efecto, además, de la caída del comercio internacional, que generaría el hundimiento de las tasas de intereses a valores negativos. Debemos normar las tasas por países emergentes, no emergentes, promoviendo el principio de transparencia. Porque su aplicación señala que tanto en los países de economía emergente y no emergente, se premia el uso eficiente de los instrumentos macroeconómicos con bajas tasas de interés, por lo contrario, altas tasas de interés a los países con elevados índices de inflación por manejo defectuoso de su economía.

Queda demostrado que, para evitar el abuso del deterioro de activos tangibles e intangibles, es propiciando tasas bajas de interés. Y su tendencia internacional futura, es hacia la baja. Si se fijan parámetros a nivel latinoamericano, como las tasas reales neutrales aquí planteadas, los Contadores Públicos en calidad de Auditores Externos, contribuirían con la obligación de hacer cumplir la norma. $\mathrm{Y}$, así por primera vez, las ciencias contables se anticiparían a los acontecimientos financieros que pueden generar los mismos efectos negativos que sucedieron en las crisis de 1929, del siglo anterior, y/o del año 2008 en el presente siglo, caracterizado por numerosas infracciones cometidas por los bancos.
El mundo está más que sorprendido con los escándalos internacionales de la corrupción. Y una medida en la dirección correcta hacia la minimización de este flagelo social, es normando las tasas de interés neutrales para efectos de la aplicación uniforme y previsible de las normas internacionales de información financiera en América Latina y el Caribe. La economía de los países y la sociedad en su conjunto, se beneficiarán al reducir la oportunidad de abusar del uso del crédito fiscal para efecto del pago de impuestos a la renta, en desmedro de los presupuestos nacionales de los países emergentes y no emergentes.

\section{REFERENCIAS BIBLIOGRÁFICAS}

Barbera, J. Gutiérrez, A. Siaba, J. (2015). Hacia una Normalización Posible. Fundación Konrad Adenauer en Argentina. Documento de Trabajo No. 95. Buenos Aires. Argentina.

Curti, D. (2010). La determinación y traspaso de las tasas de interés. Una aproximación macro bancaria. Banco Central del Uruguay. Montevideo: Uruguay.

Ferrer, A. (2010). Revisando las NIIF: NIC 36 Deterioro del Valor de los Activos. Revista "Actualidad Empresarial”, 221. Segunda Quincena de diciembre. Lima: Perú.

Ferrer, A. (2013). Revisando las NIIF: NIC 36 Deterioro del Valor de los Activos. Revista "Actualidad Empresa- 
rial”, 277. Segunda Quincena de abril. Lima: Perú.

Fondo Monetario Internacional. (2016). Perspectivas de la economía mundial: crecimiento demasiado lento por demasiado tiempo. Cuadro A6. Economías avanzadas: Precios al consumidor. Cuadro A7. Economías de mercados emergentes y en desarrollo: Precios al consumidor.

Fuentes, J.R. (2008). La tasa de interés real neutral: definiciones y evidencia para economías latinoamericanas.
Obtenida el 11 de mayo de 2017, de http://www.cemla.org/PDF/ic/2008ic/IC-05.pdf.

Gallardo, E. (2010). Evaluación del Deterioro del Valor de los Activos Fijos en una Empresa dedicada a la Fabricación de Helados. Tesis para Contador Público y Auditor. Universidad de San Carlos de Guatemala.

Gorfinkiel, D. y Lapitz, R. (2003). Globalización y Evaluación del Riesgo País. Montevideo: Uruguay.
JP Morgan. (2015) Emerging Markets Bond Index (EMBI). Washington D.C.: USA.

JP Morgan. (2016). Emerging Markets Bond Index (EMBI). Washington D.C.: USA.

Sumba D, y Sigua M. (2010). Deterioro y Baja de Activos Fijos de la Compañia de Transportes Arcentales Cía. Ltda., para el año 2010. Tesina para Contador Público Colegiado. Universidad de Cuenca. Ecuador. 